\title{
Effects of Bias Voltage on Fen Films Prepared by Magnetron Sputtering
}

\author{
Yuqiao Zeng ${ }^{a}$, Zheng Tan ${ }^{a}$, Lichu Zhou ${ }^{a}$, Meiya Jiang ${ }^{a}$,Yuedong Qiu ${ }^{a}$, Feng Fanga, Haibo Huang \\ Xuhai Zhang ${ }^{a *}$, Jianqing Jiang ${ }^{a}$ \\ a Jiangsu Key Laboratory of Advanced Metallic Materials, School of Materials Science and Engineering, \\ Southeast University, Nanjing 211189, China
}

Received: September 30, 2014; Revised: September 30, 2015

\begin{abstract}
Iron nitride films were deposited by radio frequency $(\mathrm{RF})$ reactive magnetron sputtering at different bias voltages. It was found that the composition, structure and magnetic properties of the iron nitride films were strongly dependent on the depositing bias voltage. With a bias voltage of $-50 \mathrm{~V}$, the iron nitride film is fully dense and has a homogenous microstructure consisting of $\alpha-\mathrm{Fe}$ and $\mathrm{Fe}_{16} \mathrm{~N}_{2}$, leading to a unique combination of high $M s$ of $1645 \mathrm{emu} / \mathrm{cc}, H c$ of 5 Oe and $\mu_{I}$ of 1573.
\end{abstract}

Keywords: thin film, iron nitride, magnetic sputtering, soft magnetic materials, microstructural characterization

\section{Introduction}

With the increasing requirements on power efficiency and environment protection, rare-earth free permanent magnets with high energy product are urgently demanded for future technological advances on power transmission and utilization. The iron nitride film is one of these most attractive magnet systems. In the iron nitride family, there are various phases with different lattice structures and nitrogen concentrations. Among them, the $\alpha^{\prime \prime}-\mathrm{Fe}_{16} \mathrm{~N}_{2}, \gamma^{\prime}-\mathrm{Fe}_{4} \mathrm{~N}$ and $\varepsilon-\mathrm{Fe}_{3} \mathrm{~N}$ exhibit excellent magnetic properties ${ }^{1-3}$. Therefore, a lot of works have been focused on the formation of these desirable iron nitride phases. Many growth method, such as reactive magnetron sputtering, molecular beam epitaxy, nitrogen ion implantation and etc. have been applied on different substrate to grow iron nitride films which are mainly composed of $\alpha^{\prime \prime}-\mathrm{Fe}_{16} \mathrm{~N}_{2}, \gamma^{\prime}-\mathrm{Fe}_{4} \mathrm{~N}$ ore- $\mathrm{Fe}_{3} \mathrm{~N}^{[4-6]}$. The phase evolution of the iron nitride films induced by thermal treatment was also studied. It was found that the $\alpha "-\mathrm{Fe}_{16} \mathrm{~N}_{2}$ phase is stable below $673 \mathrm{~K}$ and a slightly heated substrate will benefit the formation of $\alpha$ " $-\mathrm{Fe}_{16} \mathrm{~N}_{2}{ }^{[7,8]}$. Since the nitrogen is one of the key points to manipulate the phase compositions in the iron nitride films, the effect of nitrogen content are extensively studied ${ }^{9,10}$. However, there are few reports on the effect of bias voltage. When sputtering is used as the deposition method, bias voltage plays an important role on the composition, structure and properties of the films and coatings. For instance, the surface grain size of the Ni films on a flexible substrate increases with the increase of the bias voltage $^{11}$. When a proper negative bias was applied on the sample surface, the $\mathrm{CrSiN}$ coatings exhibit three-dimensional nanocomposite microstructure with superhigh hardness ${ }^{12,13}$.

In this work, we report the fabrication of iron nitride films with good soft-magnetic properties by changing the depositing bias voltage from $+25 \mathrm{~V}$ to $-50 \mathrm{~V}$. When a positive bias voltage is used, we intend to heat the sample surface slightly by the collision electrons which are attracted by the positive voltage. When a negative bias is used, we try to form denser structure. The relationship among the bias voltage,

*e-mail: zyuqiao@seu.edu.cn phase compositions and structure of the iron nitride films is investigated and discussed.

\section{Material and Methods}

\subsection{Film deposition}

The iron nitride films were deposited by radio frequency (RF) reactive magnetron sputtering in a mixture of Ar and $\mathrm{N}_{2}$ gases at a constant flow ratio of 30.00:0.81. A Fe target $(99.95 \%)$ with a diameter of $80 \mathrm{~mm}$ was used to provide Fe to the iron nitride films. During depositing, the working pressure was kept at $1 \mathrm{~Pa}$. The bias voltage varied from $+25 \mathrm{~V}, 0 \mathrm{~V}$ to $-50 \mathrm{~V}$ and the corresponding as-obtained films are named as film 1\#, 2\# and 3\#, respectively. (111) Si wafers was used as the deposition substrate.

The film thickness was measured by step profiler (DEKTAK 150). The chemical and phase compositions of the as-obtained iron nitride films were characterized by energy dispersive spectroscopy analyzer (EDS, FEI. Sirion 200 ) and X-ray diffraction (XRD) using $\mathrm{Cu} \mathrm{K \alpha}$ radiation, respectively. Scanning electron microscopy (SEM, FEI Sirion 200) observation was performed on the iron nitride films to investigate the surface morphologies. The more detailed microstructure of the iron nitride films was studied by using transmission electron microscopy (TEM, FEI, Tecnai G2). The hysteresis loop of the films was recorded by a vibrating sample magnetometer (VSM, Lakeshore 7404) at room temperature. Before the measurements, the VSM was calibrated by a standard Ni sample.

\section{Results}

\subsection{Formation of the iron nitride films at various bias voltages}

The thickness of the films as a function of the bias voltage is shown in Figure 1. Film 1\# has a thickness of about $584 \mathrm{~nm}$. The decrease of the bias leads to a decreasing film thickness, 
i.e. $483 \mathrm{~nm}$ at $0 \mathrm{~V}$ and $458 \mathrm{~nm}$ at $-50 \mathrm{~V}$. The most likely cause for the reduced film thickness is the decrease of macro defects such as voids or cracks. Usually the positive bias causes more macro defect sites in the functional materials and results in a loose structure. The negative bias can attract more positive ions to bombard the sample surface, leaving behind a dense structure. The electric resistance further proves the different number of macro defects of the iron nitride films deposited at different bias voltages. As shown in Figure 1, the electric resistivity of the film 1\#, 2\# and $3 \#$ is $0.161 \mathrm{ohm} / \mathrm{cm}, 0.137 \mathrm{ohm} / \mathrm{cm}$, and $0.045 \mathrm{ohm} / \mathrm{cm}$, respectively.

The chemical compositions of the iron nitride films are measured by EDS and the results are shown in Table 1. All the as-deposited samples contain both $\mathrm{Fe}$ and $\mathrm{N}$ elements, demonstrating the successful formation of iron nitride films in the depositing bias ranging from +25 to $-50 \mathrm{~V}$. The Fe/N ratio in the films keeps constant at about $12-13$ as the bias voltage decreases from $+25 \mathrm{~V}$ to $0 \mathrm{~V}$. The further decrease of the bias to $-50 \mathrm{~V}$ leads to the distinct increase of the $\mathrm{Fe} / \mathrm{N}$ ratio (about 16). Usually, when sputtering is adopted as the depositing method, more negative bias leads to more

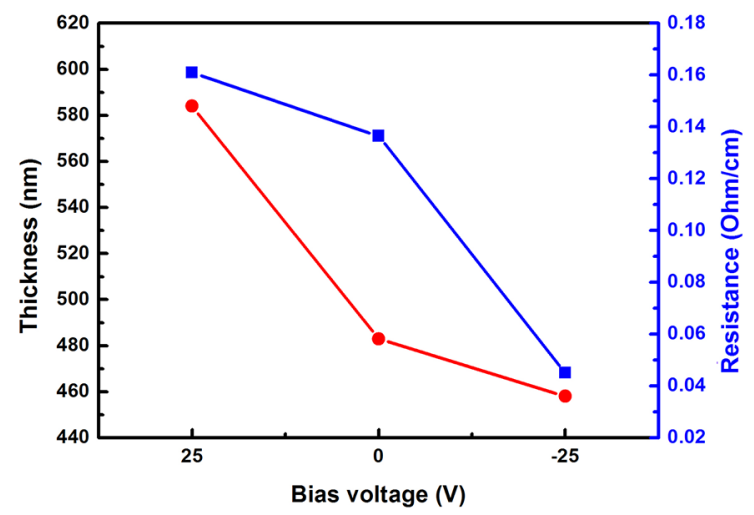

Figure 1. Thickness and the electric resistivity of the iron nitride films. reacted $\mathrm{N}$ content in the films, since the negative bias will absorb more positive $\mathrm{N}$ ions to take part into the reaction with Fe. The similar phenomenon can be observed in TiN, $\mathrm{CrN}$ films and etc. ${ }^{14,15}$. However, when the positive bias voltage is used, a loose structure with a large number of voids and cracks will form. These macro defects can absorb the gas around themselves during the deposition. Therefore the EDS tests involved some unreacted $\mathrm{N}$ elements which exist as the absorbed $\mathrm{N}_{2}$ in the iron nitride films. When the bias voltage decreases, a denser structure with less macro defects can be obtained, where the amount of absorbed $\mathrm{N}_{2}$ will decrease and lead to a lower $\mathrm{N}$ content.

As is shown in Figure 2a, film 1\# exhibits a rough surface which contains coarse pyrometric cones with a size of 30-50 nm, indicating the formation of a loose structure. The cone nature is always related to the surface diffusion process. The positive bias can attract a large number of negative electrons to the sample surface and thus enhance the local temperature on the surface. The increase of the temperature will cause higher surface diffusion rate and lead to the formation of coarse cones. Film $2 \#$ has a similar surface morphology (Figure 2b) as film 1\#. When the depositing bias decreases to a negative value, a dense structure without any cone nature is formed in the film (Figure 2c), agreeing with the results of electric resistivity.

Films $1 \#$ and $2 \#$ have a similar microstructure. In the SEAD pattern of film 2\# (Figure $3 \mathrm{a}$ ) has strong signals of the (110), (200), and (211) planes of $\alpha$-Fe. Meantime, a faint diffraction ring of the $\alpha "-\mathrm{Fe}_{16} \mathrm{~N}_{2}$ (112) can be identified.

Table 1. Chemical compositions of the iron nitride films (measured by EDS).

\begin{tabular}{cccc}
\hline Films & $\begin{array}{c}\text { Fe content } \\
\text { (at.\%) }\end{array}$ & N (at.\%) & Fe/N \\
\hline $1 \#$ & 92.3 & 7.7 & 12 \\
$2 \#$ & 92.7 & 7.3 & 13 \\
$3 \#$ & 94.2 & 5.8 & 16 \\
\hline
\end{tabular}

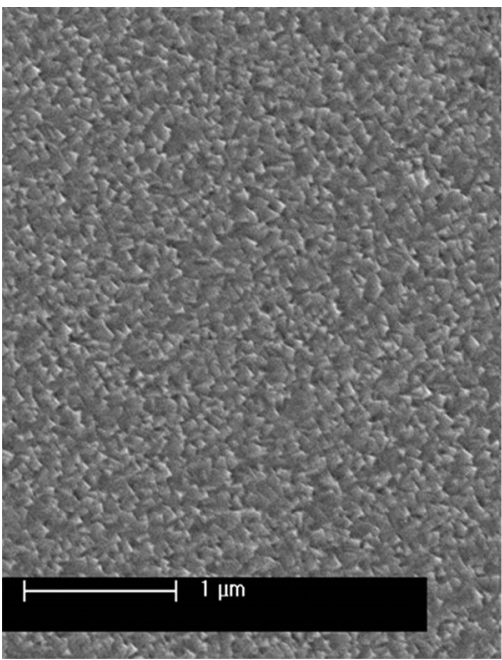

(a)

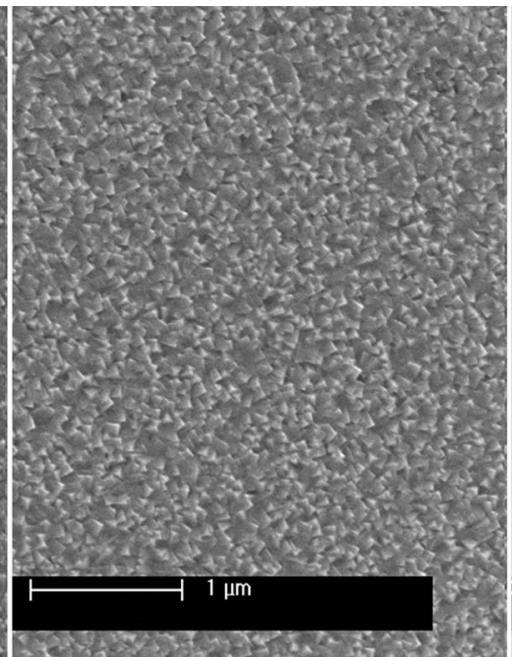

(b)

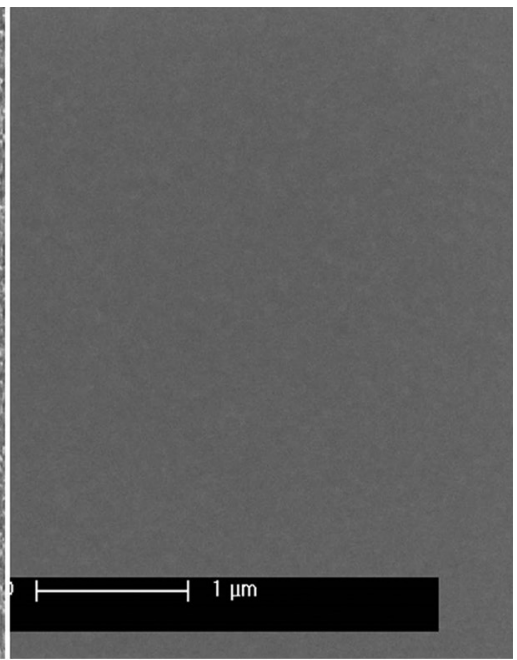

(c)

Figure 2. SEM images of the film 1\# (a), 2\# (b) and 3\# (c). 
This demonstrates the dominant growth of $\alpha$-Fe and the coexistence of a small amount of $\alpha "-\mathrm{Fe}_{16} \mathrm{~N}_{2}$ on the $\mathrm{Si}$ substrate. The bright-field TEM image in Figure $3 \mathrm{~b}$ reveals that the cone shaped crystals in film $2 \#$ have sizes in the range of 50-150 $\mathrm{nm}$. In the case of film $3 \#$, the (110), (200), and (211) planes of $\alpha$-Fe, together with the (112), (202) and (400) planes of $\alpha "-\mathrm{Fe}_{16} \mathrm{~N}_{2}$ can be identified in the SEAD patterns in Figute $3 \mathrm{c}$. The much stronger diffraction signals of the $\alpha=-\mathrm{Fe}_{16} \mathrm{~N}_{2}$ than that in Figure 3a suggests that the negative bias enhances the formation of $\alpha$ "- $\mathrm{Fe}_{16} \mathrm{~N}_{2}$ in the iron nitride films. The bright-field TEM image in Figure $3 \mathrm{~d}$ shows that film 3 \# contains a homogenous crystalline structure. The crystalline size in the direction parallel to the film surface is about $50-100 \mathrm{~nm}$. The smaller crystal size in film $3 \#$ than that in film $1 \#$ and $2 \#$ can be explained by the improved formation of the $\alpha$ " $-\mathrm{Fe}_{16} \mathrm{~N}_{2}$ in the film. The increasing amount of $\alpha "-\mathrm{Fe}_{16} \mathrm{~N}_{2}$ can provide the $\alpha$-Fe more inhomogeneous nucleation sites and retards the growth of the $\alpha$-Fe by making the atomic diffusion more difficult. As a consequence, the microstructure is refined.

The phase evolution of the iron nitride films as a function of the deposition bias is also followed by XRD in Figure 4. Film 1\#, 2\# and 3\# exhibit only one sharp peak at $2 \theta$ of about 44.58 degree, which is corresponding to the (110) of

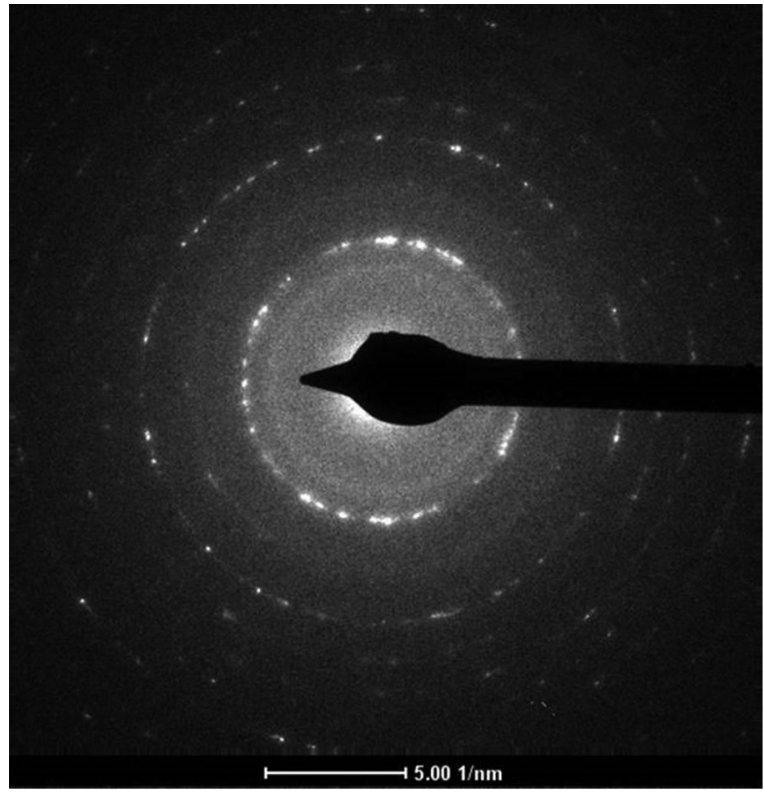

(a)

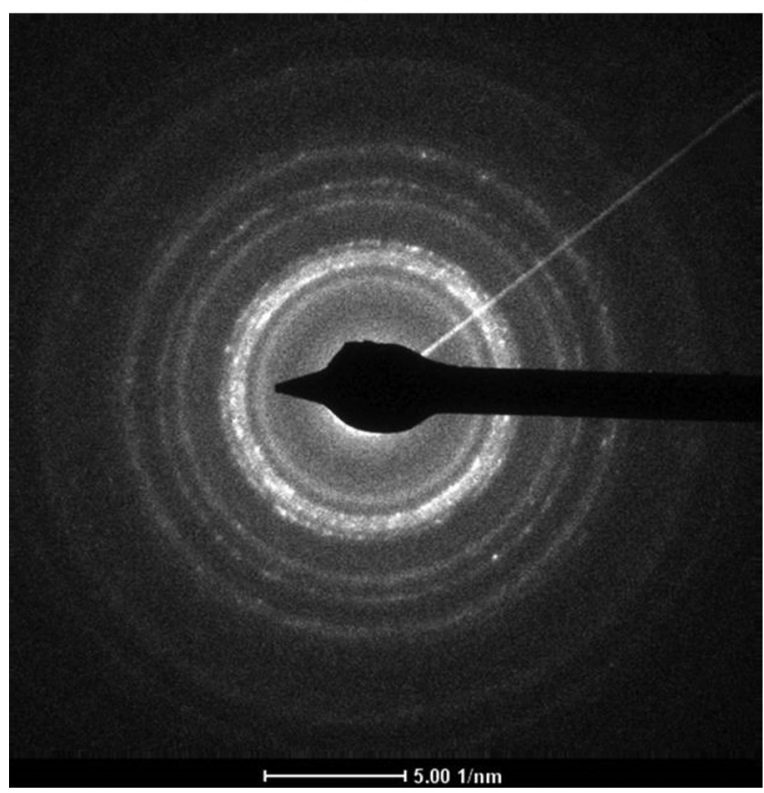

(c)

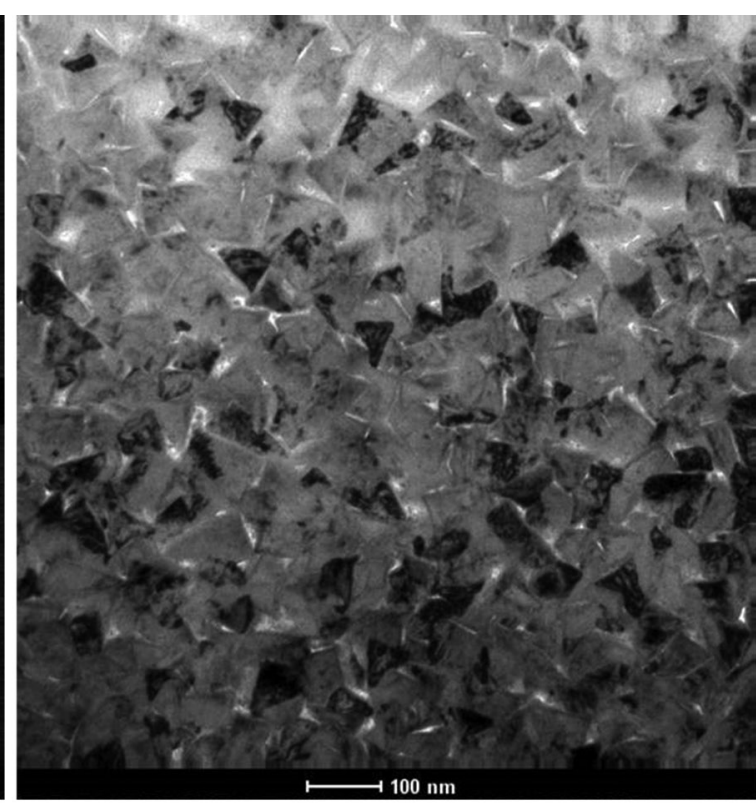

(b)

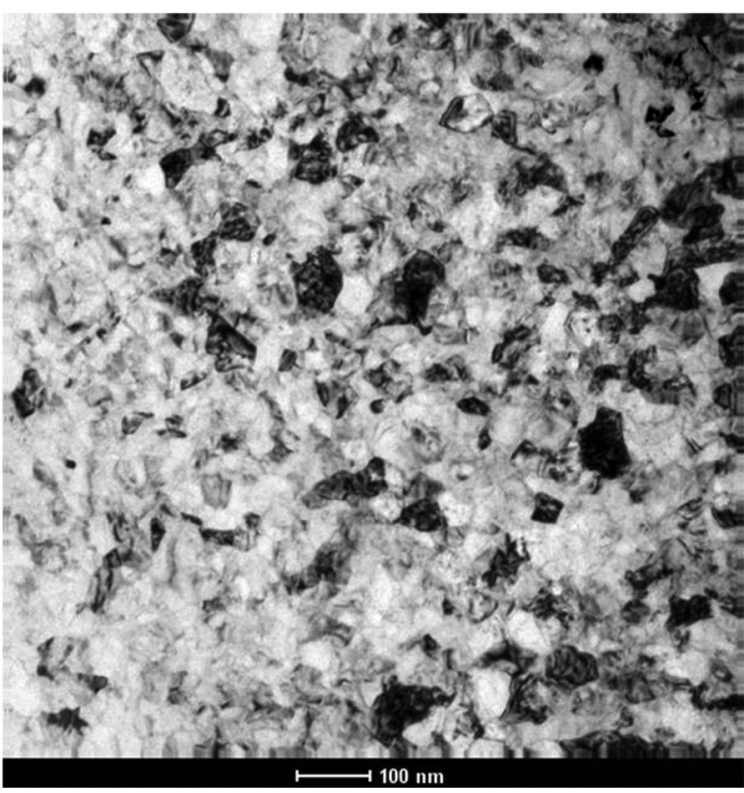

(d)

Figure 3. SEAD patterns of film 2\# (a), 3\# (c), and bright-field TEM images of film 2\# (b) and 3\# (d). 


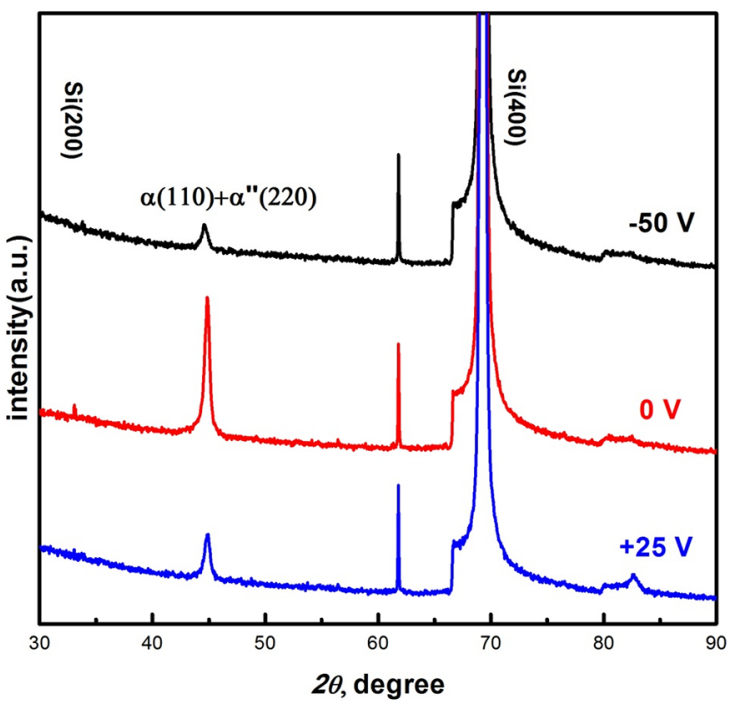

Figure 4. XRD patterns of the iron nitride films.

$\alpha$-Fe and (220) of $\alpha "-\mathrm{Fe}_{16} \mathrm{~N}_{2}$. In another words, the $\alpha$-Fe and $\alpha "-\mathrm{Fe}_{16} \mathrm{~N}_{2}$ display a preferred growth behavior according to the (110) and (220) direction, respectively. Using the Scherer formula and the profile fitting, the crystal sizes of both $\alpha$-Fe and $\alpha-\mathrm{Fe}_{16} \mathrm{~N}_{2}$ are estimated and summarized in Table 2. With the decreasing bias voltage, the $\alpha "-\mathrm{Fe}_{16} \mathrm{~N}_{2}$ is gradually refined. The $\alpha-\mathrm{Fe}$, on the contrary, gets coarsened. The results are in disagreement with the TEM observation in the plane view. However, it should be noticed that the crystal size derived from XRD data is according to the direction vertical to the sample surface. At this direction, the growth of the crystals is in a competitive way rather than though diffusion. The crystalline plane will display the higher growth rate if it contains lower chemical potential. For instance, when the N content increases, the (220) plane of TiN shows lower chemical potential than the (111) plane. The TiN will change the preferred growth direction from (111) to (200) when the $\mathrm{N}$ content is high enough ${ }^{16}$. In this study, the (111) plan of the $\alpha$-Fe may exhibit lower chemical potential than the (220) plane of the $\alpha "-\mathrm{Fe}_{16} \mathrm{~N}_{2}$ when the bias voltages decreases from $+25 \mathrm{~V}$ to $-50 \mathrm{~V}$. Thus the $\alpha$-Fe will get the champion in the growth rate competition. As a result, in the direction vertical to the sample surface, the winner has larger crystal size and the crystal structure of the loser is refined.

\subsection{Magnetic properties of the as-deposited iron nitride films}

The hysteresis loops of the iron nitride films prepared at different bias voltage is shown in Figure 5. All the iron nitride films exhibit a good magnetic softness. The detailed magnetic parameters, including $H_{C}, M_{\mathrm{s}}$ and $\mu_{I}$, are summarized in Table 3. In the case of $H_{C}$, the decreasing bias from $+25 \mathrm{~V}$ to $-50 \mathrm{~V}$ leads to a dramatic decrease of $H_{C}$ from 91 Oe to 5 Oe. On the other hand, the $M_{S}$ and $\mu_{I}$ values increase with the decrease of the bias voltage and reach their maxim value (1645 emu/cc and 1572, respectively) at $-50 \mathrm{~V}$. Thus, the iron nitride film deposited at the negative bias voltage of $50 \mathrm{~V}$ exhibit the unique combination of lowest $H_{C}$, highest $M_{S}$ and $\mu_{I}$ among the iron nitride samples deposited at different bias.

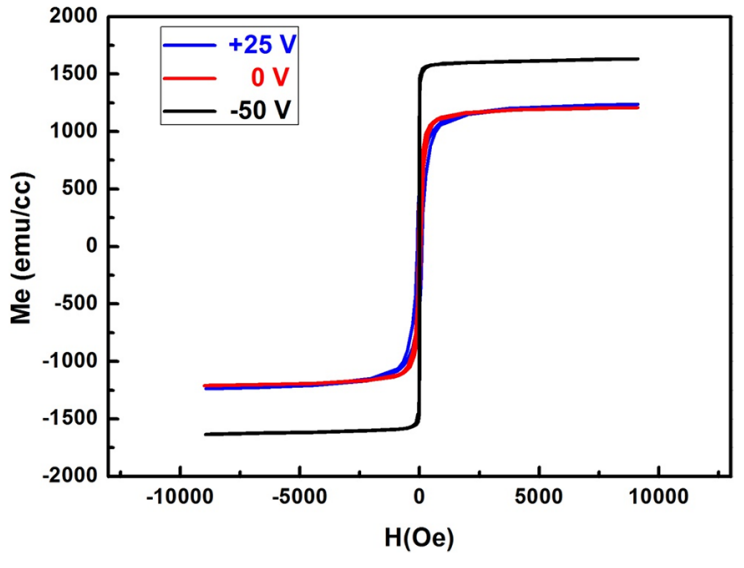

Figure 5. Hysteresis loop of the iron nitride films.

Table 2. Average grain size of $\alpha-\mathrm{Fe}$ and $\alpha "-\mathrm{Fe}_{16} \mathrm{~N}_{2}$ calculated using the Scherer formula.

\begin{tabular}{ccc}
\hline Films & $\boldsymbol{\alpha}-\mathbf{F e}(\mathbf{n m})$ & $\boldsymbol{\alpha}-\mathbf{F e}_{\mathbf{1 6}} \mathbf{N}_{\mathbf{2}}(\mathbf{n m})$ \\
\hline $1 \#$ & 18 & 46 \\
$2 \#$ & 31 & 19 \\
$3 \#$ & $>100$ & 17 \\
\hline
\end{tabular}

Table 3. Magnetic parameters of the iron nitride films.

\begin{tabular}{cccc}
\hline Films & $M_{S}(\mathbf{e m u} / \mathbf{c c})$ & $H_{C}(\mathbf{O e})$ & $\boldsymbol{\mu}_{I}$ \\
\hline $1 \#$ & 1250 & 91 & 31 \\
$2 \#$ & 1451 & 58 & 53 \\
$3 \#$ & 1645 & 5 & 1572 \\
\hline
\end{tabular}

The possible origins of the good magnetic properties may come from three aspects. The first lies in the macrostructure of the iron nitride films. The existence of macro void or cracks can be excluded by the negative bias voltage. Secondly, the crystal size matching is another important factor. The crystal size of film $3 \#$ in the direction parallel to the film surface is refined. It has been reported that the smaller crystal size in the iron nitride films is always associated with lower $H_{C}$. In the direction vertical to the film surface, film $3 \#$ contains larger $\alpha$-Fe than the other films. Larger crystal size can benefit the $M_{S}$ by reduce the spin disorder. Thirdly, it has been well documented that the phase composition is a key point to the magnetic properties. Since $\alpha "-\mathrm{Fe}_{16} \mathrm{~N}_{2}$ exhibit much higher $M_{S}$ than $\alpha-\mathrm{Fe}$, the higher $\alpha$ " $-\mathrm{Fe}_{16} \mathrm{~N}_{2}$ content in the iron nitride films will result in higher $M_{S}$.

\section{Conclusions}

A series of iron nitride films were deposited by RF reactive magnetron sputtering at various bias voltages. It was found that the composition, structure and magnetic properties of the iron nitride films were strongly depended on the depositing bias voltage. The bias of $-50 \mathrm{~V}$ led to a unique combination of high $M_{S}$ of $1645 \mathrm{emu} / \mathrm{cc}, H_{C}$ of $5 \mathrm{Oe}$ and $\mu_{I}$ of 1573. A homogenous dense microstrure composed of $\alpha$-Fe and $\alpha$ " $-\mathrm{Fe}_{16} \mathrm{~N}_{2}$ might be attributed to the high soft magnetic performance of the iron nitride film prepared at $-50 \mathrm{~V}$. 


\section{Acknowledgements}

This work is supported by the Fundamental Research Funds for the Central Universities, National Science Foundation of China (51141004, 51201033), Specialized Research Fund for the Doctoral Program of Higher

\section{References}

1. Kim TK and Takahasi M. New magnetic material having ultrahigh magnetic moment. Applied Physics Letters. 1972; 20(12):492-494. http://dx.doi.org/10.1063/1.1654030.

2. Takahashi T, Takahashi $\mathrm{N}$ and Nakamura T. Preparation of freestanding $\mathrm{Fe}_{2} \mathrm{~N}$ crystal by vapor-phase epitaxy under atmospheric pressure. Materials Chemistry and Physics. 2004; 83(1):7-9. http://dx.doi.org/10.1016/j.matchemphys.2003.08.028.

3. Chen GM, Jaggi NK, Butt JB, Yeh EB and Schwartz LH. Mossbauer and magnetic studies of $\varepsilon-\mathrm{Fe}_{\mathrm{x}} \mathrm{N}, 2<\mathrm{x} \leq 3$. Journal of Physical Chemistry. 1983; 87(26):536-5332. http://dx.doi. org/10.1021/j150644a005.

4. Feng XP, Mi WB and Bai HL. Investigation of structure and magnetic properties of the as-deposited and post annealed iron nitride films by reactive facing-target sputtering. Applied Surface Science. 2011; 257(16):7320-7325. http://dx.doi.org/10.1016/j. apsusc.2011.03.112.

5. Sugita Y, Mitsuoka K, Komuro M, Hoshiya H, Kozono Y and Hanazono M. Giant magnetic moment and other magnetic properties of epitaxially grown $\mathrm{Fe}_{16} \mathrm{~N}_{2}$ single-crystal films. Journal of Applied Physics. 1991; 70(10):5977-5982. http:// dx.doi.org/10.1063/1.350067.

6. Jiang YF, Al Mehedi M, Fu EG, Wang YQ and Wang JP. FeN foils by nitrogen ion-implantation. Journal of Applied Physics. 2014; 115(17):17A753.

7. Terada N, Hoshi Y, Naoe M and Yamanaka S. Synthesis of iron-nitride films by means of ion beam deposition. IEEE Transactions on Magnetics. 1984; 20(5):1451-1453. http:// dx.doi.org/10.1109/TMAG.1984.1063545.

8. Nakajima K, Yamashita T, Takata M and Okamoto S. Mobbauer study on $\mathrm{Fe}_{16} \mathrm{~N}_{2}$ films prepped by ion-implant nitrification of iron films. Journal of Applied Physics. 1991; 70(10):6033-6035. http://dx.doi.org/10.1063/1.350084.

9. Morisako A, Takahashi K, Matsumoto M and Naoe M. Iron nitride thin-films prepared by facing targets sputtering. Journal
Education (20110092120035), Opening Project of Jiangsu Key Laboratory of Advanced Metallic Materials (AMM201101), Opening Project of Jiangsu Key Laboratory of Material Tribology (kjsmcx1003), and Fundamental Research Funds for the Central Universities (3212003101, 3212002205, 3212003102).

of Applied Physics. 1988; 63(8):3230-3232. http://dx.doi. org/10.1063/1.340851.

10. Mi WB, Feng XP and Bai HL. Magnetic properties and Hall effect of reactive sputtered iron nitride nanocrystalline films. Journal of Magnetism and Magnetic Materials. 2011; 323(14):19091913. http://dx.doi.org/10.1016/j.jmmm.2011.02.043.

11. Koda $\mathrm{T}$ and Toyota $\mathrm{H}$. Effects of magnetic flux density and substrate bias voltage on $\mathrm{Ni}$ films prepared on a flexible substrate material using unbalanced magnetron sputtering assisted by inductively coupled plasma. Journal of Vaccum Science \& Technology A. 2014; 32(2):02B104.

12. Wang QM and Kim K. Microstructural control of $\mathrm{Cr}-\mathrm{Si}-\mathrm{N}$ films by a hybrid arc ion plating and magnetron sputtering process. Acta Materialia. 2009; 57(17):4974-4987. http:// dx.doi.org/10.1016/j.actamat.2009.07.001.

13. Lin J, Wang B, Ou Y, Sproul W, Dahan I and Moore J. Structure and properties of $\mathrm{CrSiN}$ nanocomposite coatings deposited by hybrid modulated pulsed power and pulsed dc magnetron sputtering. Surface and Coatings Technology. 2013; 216:251258. http://dx.doi.org/10.1016/j.surfcoat.2012.11.053.

14. Kong Q, Ji L, Li H, Liu X, Wang Y, Chen J, et al. Influence of substrate bias voltage on the microstructure and residual stress of $\mathrm{CrN}$ films deposited by medium frequency magnetron sputtering. Materials Science and Engineering B - Advanced Functional Solid-State Materials. 2011; 176(11):850-854.

15. Wang Z, Zhang D, Ke P, Liu X and Wang A. Influence of substrate negative bias on structure and properties of TiN coatings prepared by hybrid HIPIMS method. Journal of Materials Science and Technology. 2015; 31(1):37-42. http:// dx.doi.org/10.1016/j.jmst.2014.06.002

16. Gall D, Kodambaka S, Wall M, Petrov I and Greene JE. Pathways of atomistic processes on TiN(001) and (111) surfaces during film growth: an ab initio study. Journal of Applied Physics. 2003; 93(11):9086-9095. http://dx.doi.org/10.1063/1.1567797. 